\title{
The Mass of the Central Black Hole in the Seyfert Galaxy NGC 3783
}

\author{
Christopher A. Onken \& Bradley M. Peterson \\ Department of Astronomy, The Ohio State University, Columbus, OH 43210 \\ onken, peterson@astronomy.ohio-state.edu
}

\begin{abstract}
Improved analysis of ultraviolet and optical monitoring data on the Seyfert 1 galaxy NGC 3783 provides evidence for the existence of a supermassive, $(8.7 \pm 1.1) \times 10^{6} \mathrm{M}_{\odot}$, black hole in this galaxy. By using recalibrated spectra from the International Ultraviolet Explorer satellite and ground-based optical data, as well as refined techniques of reverberation mapping analysis, we have reduced the statistical uncertainties in the response of the emission lines to variations in the ionizing continuum. The different time lags in the emission line responses indicate a stratification in the ionization structure of the broad-line region and are consistent with the virial relationship suggested by the analysis of similar active galaxies.
\end{abstract}

Subject headings: galaxies: active — galaxies: individual (NGC 3783) — galaxies: nuclei — galaxies: Seyfert - ultraviolet: galaxies

\section{INTRODUCTION}

The primary model that has emerged over the last few decades for the radiation source of an active galactic nucleus (AGN) is accretion onto a supermassive black hole (SMBH). Variations in the ionizing continuum have been seen to influence the strength of emission lines arising from the broad-line region (BLR). Cross-correlation of the continuum and emission line light curves yields a characteristic time lag with which each line echoes the continuum fluctuations (Blandford \& McKee 1982). This reverberation mapping technique has been used to measure the sizes of BLRs for a growing number of AGNs (see Wandel, Peterson, \& Malkan 1999; Kaspi et al. 2000).

In addition to the BLR size, reverberation analysis can be used to estimate the mass of the SMBH. The reliability of these reverberation masses has been debated because of the uncertainty surrounding the common assumption of virialized BLR gas motions. The detailed kinematics and structure of the BLR is an unresolved issue and could lead to systematic errors on the order of a factor of a few or perhaps more (see
Fromerth \& Melia 2001; Krolik 2001). However, the excellent agreement between the black hole mass-bulge velocity dispersion (M- $\sigma$ ) relationships for reverberation-mapped AGNs and normal galaxies (Ferrarese et al. 2001) suggests the systematic discrepancy introduced in reverberation mapping is small. Additionally, AGNs for which multiple emission lines have been mapped (NGC 5548, 3C 390.3, NGC 7469) show an inverse relationship between the time lag and the emission line width, consistent with the gas motions being dominated by the gravity of the SMBH (Peterson \& Wandel 1999, 2000).

A combined optical and UV monitoring campaign was carried out on the Seyfert 1 galaxy NGC 3783 by the International AGN Watch consortium, making use of the International Ultraviolet Explorer (IUE), the Hubble Space Telescope, and a host of ground-based observatories over a period of 7 months in 1991-1992. The results of that work have been published by Reichert et al. (1994), Stirpe et al. (1994), and Alloin et al. (1995). Compared to the consortium's earlier study of another Seyfert galaxy, NGC 5548 (see Clavel et al. 1991; Peterson et al. 1991; Dietrich et al. 1993), the 
emission-line time lags were relatively uncertain, too poorly constrained in fact to reveal any possible virial relationship between line width and time lag.

The continued rarity of such campaigns, however, makes it clearly desirable to learn as much as possible from the extant datasets. This provides the motivation for our current study.

With the release of an updated processing pipeline and calibration for IUE data, the possibility arose to re-analyze the spectra of NGC 3783 and reduce the uncertainties of the emission-line time lags. In addition, the techniques of reverberation analysis have matured in the years since the original data were published, now providing more consistent methodology for cross-correlation and error estimation. Thus, we have re-examined the data, deriving more precise results for the emission line reverberation and revising the previous estimates of the reverberation mass.

The next section describes the observations and how the data were reduced ( $§ 2)$. In $\S 3$ we explain the analysis procedure and give our crosscorrelation results. Section 4 discusses the results and the SMBH mass determination, and our conclusions are summarized in $\S 5$.

\section{OBSERVATIONS AND DATA RE- DUCTION}

\subsection{UV Data}

The IUE observations of NGC 3783 were conducted in 69 separate epochs, with two sampling rates. The first interval (of 45 epochs) had an average spacing of 4.0 days, while the final 24 epochs observed the AGN with an average spacing of 2.0 days. A more complete description of the UV observing program is provided by Reichert et al. (1994).

In addition to the original IUE Spectral Image Processing System (IUESIPS), Reichert et al. (1994) used a Gaussian extraction method (GEX; see Clavel et al. 1991) to obtain the spectra of NGC 3783. After the original data had been taken, a new standard processing pipeline was introduced. The main advantages of the New Spectral Image Processing System (NEWSIPS; Nichols et al. 1993) with respect to the older IUESIPS are the improved photometric accuracy and higher
$S / N$ of the spectra; these characteristics have been achieved by introducing a new method of raw data science registration (which both reduces the fixed pattern noise in the images and improves the photometric corrections), a weighted slit extraction method, and re-derived absolute flux calibrations. NEWSIPS also includes corrections for nonlinearity that might have affected previous studies. Overall, NEWSIPS-processed spectra show average $S / N$ increases of $10-50 \%$ over IUESIPS data (Nichols \& Linsky 1996).

We retrieved the NEWSIPS-extracted short wavelength prime camera (SWP; Harris \& Sonneborn 1987) spectra from the IUE Final Archive ${ }^{1}$. While Reichert et al. (1994) analyzed data from both the SWP and long wavelength prime cameras, we have limited our study to observations made with the SWP instrument, which has a wavelength range of $1150-1975 \AA$ in the lowdispersion mode (Newmark et al. 1992).

Each spectrum was examined and several types of problems led to spectra being removed from further consideration: (1) low $S / N$ (determined by inspection, but corresponding roughly to a continuum $S / N$ limit of 10); (2) unusual spectral features (possibly due to grazing cosmic-ray impacts); (3) short exposure times (when longerexposure data were available from the same epoch and the line flux data were discrepant). Some anomalous features were checked against the GEX frames, from which cosmic ray impacts were carefully removed. Problems with the spectra were ignored in cases where they occurred in spectral regions outside those used in computing line and continuum fluxes. Continuum and emission line flux values were measured using the wavelengths limits listed in Table 1.

The continuum was defined by a linear fit through four spectral regions (1340-1370 A, 1440$1480 \AA, 1710-1730 \AA$, and 1840-1860 ̊). An alternate fit through the first three of these regions produced consistent results. Wavelength-specific problems in two cases (SWP 45150, SWP 45206) led us to substitute the alternate continuum fit for these spectra.

We have estimated the flux uncertainties by considering instances in which multiple independent exposures were obtained at the same epoch

\footnotetext{
${ }^{1}$ http://ines.laeff.esa.es/ines/
} 
(i.e., a single pointing toward the target). Flux ratios between pairs of points within each epoch were calculated and the standard deviation of the flux ratios was taken as the fractional uncertainty for all observations. This analysis was conducted independently for each emission line and continuum band. As noted above, however, highly discrepant data were removed prior to this analysis. In spite of our use of an edited dataset, the large number of data pairs contributing to our error estimate (about 35) justifies our continued use of these values in the analysis. The final UV dataset is given in Table 2 for the continuum measurements and in Table 3 for the emission lines.

The velocity width desired for the reverberation mass calculation is related to the emission line velocity full width at half-maximum $\left(V_{F W H M}\right)$ by

$$
\sigma=\frac{\sqrt{3} V_{F W H M}}{2}
$$

where the factor of $\sqrt{3} / 2$ is used to maintain consistency with previous work (e.g., Wandel et al. 1999; Kaspi et al. 2000) and assumes isotropic gas motion.

An RMS spectrum was created from the data to isolate the varying parts of the emission lines and it was from this spectrum that the primary $V_{F W H M}$ values for the emission lines were measured. The $V_{F W H M}$ data were constructed by considering the extreme flux values within the continuum regions, fitting two continuum slopes (to the highest flux levels and lowest flux levels), and averaging the measures of $V_{F W H M}$ derived from the two continuum determinations. Finally, the data were converted to their rest-frame widths using $z=0.009730 \pm 0.000007$ (Theureau et al. 1998). Previous work examining the difference between using the mean and RMS spectra have not produced significantly different results (e.g., Kaspi et al. 2000), but in principle the RMS spectrum should better trace the gas with which we are concerned. We have measured line widths from both spectra (Figure 1) and report the results of our mean and RMS $V_{F W H M}^{\text {rest }}$ measurements in Table 4. Geocoronal Ly $\alpha$ emission blended into the Ly $\alpha$ spectral region precludes $V_{F W H M}$ measurement for this line and thus also prevents Ly $\alpha$ contribution to the mass determination, but crosscorrelation analysis is still feasible by excluding the contaminated portion of the spectrum (see Ta- ble 1).

\subsection{Optical Data}

Ground-based optical spectroscopy was conducted over the same time period as the IUE observations. The optical data analyzed here were retrieved from the $A G N$ Watch website ${ }^{2}$, and details of the observations are described by Stirpe et al. (1994). We have limited our investigation to the data gathered at the Cerro Tololo InterAmerican Observatory (CTIO) $1.0 \mathrm{~m}$ telescope to ensure the most homogeneous dataset possible for the cross-correlation analysis and for the construction of the mean and RMS spectra. Spectra that were excessively noisy or contained other anomalies were discarded from consideration, leaving 37 CTIO observations for further analysis.

Narrow spectral lines are assumed not to vary over the timescales these data are probing. Thus the individual spectra were scaled to a constant flux by using the spectral scaling technique of van Groningen \& Wanders (1992). This method computes a smooth scaling function between the input spectrum and a reference (the mean spectrum in this case, shown in Figure 2) over a specified wavelength range. We scaled over the spectral region $4972-5150 \AA$ in order to span the redshifted [O III] $\lambda \lambda 4959,5007$ emission lines and a suitable amount of continuum. We found that two iterations were required for full convergence. This reduced the fractional RMS scatter in the [O III] $\lambda 5007$ light curve (measured between 5028 and $5090 \AA$ ) to less than $2.5 \%$. Additional iterations failed to produce any light curves with smaller scatter. The mean [O III] $\lambda 5007$ flux was normalized to $8.44 \times 10^{-13}$ erg $\mathrm{s}^{-1} \mathrm{~cm}^{-2}$, the value derived by the careful analysis of Stirpe et al. (1994).

Following the calibration of the spectra, the $\mathrm{H} \beta$ line was measured between 4830 and $4985 \AA$ (with the continuum set by a linear fit between 4800 $4820 \AA$ and 5130-5150 ̊). The flux uncertainties were measured in the same way as for the UV data (§2.1), and the results are given in Table 5. Due to the smaller optical dataset, the flux errors for $\mathrm{H} \beta$ and the $5150 \AA$ continuum rely on only seven data pairs. To be cautious, we have been more conservative in our estimation of the optical flux uncertainties. The method for measuring $V_{F W H M}^{r e s t}$ was

\footnotetext{
${ }^{2}$ http://www.astronomy.ohio-state.edu/ agnwatch/
} 
also applied to the optical data and yielded values of $(2.91 \pm 0.19) \times 10^{3} \mathrm{~km} \mathrm{~s}^{-1}$ for the RMS spectrum and $(2.65 \pm 0.02) \times 10^{3} \mathrm{~km} \mathrm{~s}^{-1}$ for the mean optical spectrum.

\section{LIGHT CURVE ANALYSIS}

In Table 6 we compare the sampling characteristics of our data with the previously published light curves. When we bin the data in each epoch, the variability parameters of the old and new UV datasets appear nearly identical. The "excess variance", $F_{v a r}$, represents the mean fractional variation of each dataset (see Rodrígues-Pascual et al. $1997) ; R_{\max }$ is the ratio of maximum to minimum flux levels. The updated optical dataset is much more sparse than the previously published data because of our desire for the most homogeneous dataset possible.

Figure 3 shows the light curves for each of the UV and optical emission lines and continuum bands. Applying the techniques described by Peterson et al. (1998), we generated cross-correlation functions (CCFs) relating the various emission line light curves to the $1355 \AA$ continuum flux. We report both peak $\left(\tau_{\text {peak }}\right)$ and centroid $\left(\tau_{\text {cent }}\right)$ crosscorrelation lags. However, the reader should be warned that "lags" in the text will hereafter refer to centroids, unless otherwise noted, and that such lags do not represent a simple phase shift between the light curves.

As Koratkar \& Gaskell (1991a) noted for NGC 3783 (and other reverberation-mapped AGNs), the choice of what threshold to use for the centroid calculation can significantly affect the resulting time lag. Figure 4 shows that some lines tend toward larger lags and others toward smaller values as the centroid becomes increasingly dominated by the peak value. For the interpolated $\mathrm{CCF}$ (ICCF; Gaskell \& Peterson 1987; White \& Peterson 1994), we experimented with different interpolation lengths and different thresholds for the calculation of the lag centroid. Our subsequent analysis uses an interpolation unit of 0.1 days in both light curves (interpolating one dataset at a time, with the resulting lags averaged) and a centroid threshold of $80 \%$ of the peak correlation coefficient.

In addition to the ICCF, we calculated the discrete correlation function (DCF; Edelson \& Kro- lik 1988) for each continuum band and emission line. While the DCF, which requires binning of the data, is more likely to miss a real correlation than the ICCF under poor sampling conditions, it is also less likely to introduce a spurious relationship (White \& Peterson 1994). Gaskell (1994) notes that the DCF also relies on interpolation, but does so in the correlation function, rather than the original time series. The ICCF and DCF methods have been compared by various authors (e.g., White \& Peterson 1994; Litchfield, Robson, \& Hughes 1995) and typically yield similar results.

To assess the uncertainties in the time lag calculations, we used the Monte Carlo (MC) methods of Peterson et al. (1998). This technique for modelindependent error estimation consists of two components, each testing for a separate contribution to the cross-correlation uncertainty. To account for the uncertainty in an individual flux measurement, each data point in the light curve is altered by a random Gaussian deviation that corresponds to the quoted flux error (calculated by the method described in $\S 2.1$ ). The result of many such realizations, referred to as "flux randomization" (FR), should yield average values equal to the original data with standard deviations given by the original uncertainties. Secondly, the effects of nonuniform temporal sampling of the AGN fluctuations are investigated with "random subset selection" (RSS). Given a sample of $N$ observations, $N$ data points are randomly chosen from the set (ignoring whether they have been chosen previously). While DCF and ZDCF (Alexander 1997) analyses can weight multiply-selected data, the ICCF (which we use for our MC calculations) does not consider the flux uncertainties and simply excludes the redundant data points. Ignoring these data reduces the set by $\sim N / e$ on average and so should yield a wider range of peak lags from the ICCF. Repeated MC realizations (at least $10^{3}$ in the present work; combining the FR/RSS methods for each calculation) are used to create a cross-correlation peak distribution (CCPD; Maoz \& Netzer 1989), which provides an empirical measurement of the uncertainties for both $\tau_{\text {cent }}$ and $\tau_{\text {peak }}$.

\subsection{Emission Lines}

The light curves for each emission line (He II $\lambda 1640+$ O III] $\lambda 1663$, Si IV $\lambda 1400+$ O IV] $\lambda 1402$, 
Ly $\alpha$, C IV $\lambda 1549$, Si III] $\lambda 1892+$ C III] $\lambda 1909$, and $\mathrm{H} \beta$ ) were run through the ICCF, DCF, and FR/RSS programs, using the $1355 \AA$ continuum data as the "driving" light curve. The CCFs and CCPDs are shown for each emission line in the panels of Figure 5. The CCPDs are shown to give a graphical indication of the empirical uncertainties and are scaled to the maximum value in each panel.

Each of the emission lines was very well correlated with the continuum flux. The poorest correlation with the continuum was found for Si III] $\lambda 1892+$ C III $] \lambda 1909$, which was found to have a peak ICCF value of $\mathrm{r}_{\max }=0.354$ (i.e., a probability of arising from an uncorrelated parent population of roughly $<0.001$ ) and we limited the range of computation for this line to \pm 16 days to avoid aliasing. Table 7 summarizes the previous data and our new results.

Our results for the UV emission lines are generally in agreement with those of Reichert et al. (1994). It should be noted, however, that the peak and centroid lags calculated by Reichert et al. (1994) and Stirpe et al. (1994) used the $1460 \AA$ continuum as the driving light curve. The results quoted here are consistent with those derived from the recalibrated data with the continuum centered at $1460 \AA$ rather than at $1355 \AA$. Because of the large uncertainties assigned to previous lag values, most of our NEWSIPS lags are within $1-\sigma$ of the old data. The exceptions are Si III] $\lambda 1892+$ C III] $\lambda 1909$, for which the IUESIPS-based data failed to produce any lag at all, and $\mathrm{H} \beta$. The GEX extraction method yielded a peak lag for Si III] $\lambda 1892+$ C III] $\lambda 1909$ similar to what we found, but a centroid lag approximately $2-\sigma$ larger than the current result. Our centroid lag for $\mathrm{H} \beta$ was only slightly more than $1-\sigma$ greater than the previous value.

The significant discrepancy between our $\mathrm{H} \beta$ results and those of Wandel et al. $\left(1999,4.5_{-3.1}^{+3.6}\right.$ days) arises from the double-peaked nature of the $\mathrm{CCF}$. The centroid lag calculated for the $5150 \AA$ continuum- $\mathrm{H} \beta \mathrm{CCF}$ is based on fewer points than the $1355 \AA$ continuum- $\mathrm{H} \beta$ lag, and gives precedence to the peak at smaller lags. We have greater confidence in the results that use the UV continuum data, and those results closely match the UV$\mathrm{H} \beta$ correlation found by Stirpe et al. (1994).

\subsection{Continuum}

Strong evidence for wavelength-dependent continuum lags has been found for only two AGNs (NGC 7469 and Akn 564), but appears to be consistent with simple accretion disk models that predict $\tau \propto \lambda^{4 / 3}$ (see Wanders et al. 1997; Collier et al. 1998, 2001). However, Korista \& Goad (2001) note that diffuse emission from broad-line clouds can produce a similar wavelength dependence, so the origin of this phenomenon is not clear.

The large uncertainties still present in the NEWSIPS continuum lags prevent us from reasonably testing the $\tau-\lambda$ relationship because the continuum-continuum time lags we find are not statistically significant (see Table 7 ).

\section{IMPLICATIONS FOR THE BLR AND THE SMBH}

As the tabular data indicate, the expected pattern of more highly ionized lines having smaller time lags (i.e., originating closer to the ionization source) is reconfirmed by our analysis.

Figure 6 plots $V_{F W H M}^{\text {rest }}(R M S)$ versus $\tau_{\text {cent }}^{\text {rest }}$ for the five emission lines we measured. The virial assumption predicts a slope of -0.5 (in $\log -\log$ space). Deviation from this relationship would contradict our model, but agreement with the predicted slope cannot rule out other dynamical possibilites (see Krolik 2001, and references therein).

The statistical problem of fitting to intrinsically scattered data with heteroscedastic errors has been addressed with computational methods by Akritas \& Bershady (1996). However, our data has the additional difficulty of asymmetric errors in the lags. To account for the asymmetric time lag uncertainties we first used the larger of the two lag errors and then assessed in which direction the data points differed from the regression. We recalculated the fit using the errors toward the previous regression and confirmed that those were the appropriate choices in the final fit. The slope of the $V_{F W H M}^{\text {rest }}(R M S)-\tau_{\text {cent }}^{\text {rest }}$ relation derived by the regression software ${ }^{3}$ was $-0.450 \pm 0.070$, consistent with our expectations for a virial relationship (irrespective of the specific multiplicative factor relating the line widths and $V_{F W H M}$ values). Hence, we fixed the slope at -0.5 and calculated the mass

\footnotetext{
${ }^{3}$ available at http://www.astro.wisc.edu/ $\sim$ mab/archive/stats/stats.html
} 
independently for each emission line, applying our previously stated assumption of isotropic BLR gas motion and inserting the appropriate rest-frame values into the following equation:

$$
M=\frac{3 c \tau V_{F W H M}^{2}}{4 G} .
$$

Weighting the data by the uncertainty in the direction of the mean (since the lag errors are still asymmetric) yields an average SMBH mass of $(8.7 \pm 1.1) \times 10^{6} \mathrm{M}_{\odot}$.

Previous work with IUE archival data having much poorer temporal resolution measured a much larger C IV $\lambda 1549$ time lag and derived a mass of $7.3_{-3.6}^{+3.5} \times 10^{7} \mathrm{M}_{\odot}$ (Koratkar \& Gaskell 1991a,b). Wandel et al. (1999) calculated the $\mathrm{H} \beta$ lag with respect to the $5100 \AA$ continuum from the light curves of Stirpe et al. (1994) and then used the RMS velocity width to estimate a mass of $1.1_{-1.0}^{+1.1} \times 10^{7} \mathrm{M}_{\odot}$. Applying this method to our version of the optical data yields an SMBH mass of $6.2_{-6.1}^{+4.7} \times 10^{6} M_{\odot}$, within the $1-\sigma$ error bars for our mass measurement with the full dataset. Fromerth \& Melia (2000) employed a different means of measuring the velocity dispersion from the data of Reichert et al. (1994) and derived masses of $1.6_{-0.4}^{+0.8} \times 10^{7} \mathrm{M}_{\odot}$ and $1.3_{-0.5}^{+0.8} \times 10^{7} \mathrm{M}_{\odot}$ from Ly $\alpha$ and C IV $\lambda 1549$, respectively. Various disk accretion models predicting a SMBH mass in the range of $2.0-7.0 \times 10^{7} \mathrm{M}_{\odot}$ were cited by Alloin et al. (1995). However, they note the simple nature of these spatially thin, optically thick disk models and the potential for a large discrepancy from the true SMBH mass.

\section{SUMMARY}

We have conducted reverberation mapping analysis on recalibrated IUE and ground-based optical observations of the Seyfert 1 galaxy NGC 3783 with the goal of revising the mass estimate for the central SMBH. The NEWSIPS spectra confirm the existence of varying time lags for emission lines of different ionization potentials and provide a better constraint on the SMBH mass under the assumption of virial gas motion. The emission line time lags vary from 1.3 to 10.4 days, and analysis of peak and centroid time lags yield similar results for each line. Our mass determination revises the previous values to a mass of $(8.7 \pm 1.1) \times 10^{6} \mathrm{M}_{\odot}$.
We gratefully acknowledge support for this work through NASA grant NAG5-8397. C. A. O. thanks The Ohio State University for support through the Distinguished University Fellowship. We also thank Patrizia Romano for acquiring the NEWSIPS data, and Matthew A. Bershady for making available his statistical software. This research has made use of the NASA/IPAC Extragalactic Database (NED) which is operated by the Jet Propulsion Laboratory, California Institute of Technology, under contract with the National Aeronautics and Space Administration.

\section{REFERENCES}

Akritas, M. G. \& Bershady, M. A. 1996, ApJ, 470, 706

Alexander, T. 1997, in Astronomical Time Series, ed. D. Maoz, A. Sternberg, \& E. M. Leibowitz (Dordrecht: Kluwer), 163

Alloin, D., et al. 1995, A\&A, 293, 293

Blandford, R. D., \& McKee, C. F. 1982, ApJ, 255, 419

Clavel, J., et al. 1991, ApJ, 366, 64

Collier, S. J., et al. 1998, ApJ, 500, 162

Collier, S., et al. 2001, ApJ, 561, 146

Dietrich, M., et al. 1993, ApJ, 408, 416

Edelson, R. A., \& Krolik, J. H. 1988, ApJ, 333, 646

Ferrarese, L., Pogge, R. W., Peterson, B. M., Merritt, D., Wandel, A., \& Joseph, C. L. 2001, ApJ, 555, L79

Fromerth, M. J., \& Melia, F. 2000, ApJ, 533, 172

Fromerth, M. J., \& Melia, F. 2001, in ASP Conf. Ser. 224, Probing The Physics Of Active Galactic Nuclei By Multiwavelength Monitoring, ed. B. M. Peterson, R. S. Polidan, \& R. W. Pogge (San Francisco: ASP), 377

Gaskell, C. M. 1994, in ASP Conf. Ser. 69, Reverberation Mapping Of The Broad-Line Region In Active Galactic Nuclei, ed. P. M. Gondhalekar, K. Horne, \& B. M. Peterson (San Francisco: ASP), 111 
Gaskell, C. M., \& Peterson, B. M. 1987, ApJS, 65, 1

Harris, A. W., \& Sonneborn, G. 1987, in Exploring the Universe with the IUE Satellite, ed. Y. Kondo (Dordrecht: Reidel), 729

Kaspi, S., Smith, P. S., Netzer, H., Maoz, D., Jannuzi, B. T., \& Giveon, U. 2000, ApJ, 533, 631

Koratkar, A. P., \& Gaskell, C. M. 1991a, ApJS, 75,719

Koratkar, A. P., \& Gaskell, C. M. 1991b, ApJ, 370, L61

Korista, K. T., \& Goad, M. R. 2001, in ASP Conf. Ser. 224, Probing The Physics Of Active Galactic Nuclei By Multiwavelength Monitoring, ed. B. M. Peterson, R. S. Polidan, \& R. W. Pogge (San Francisco: ASP), 411

Krolik, J. H. 2001, ApJ, 551, 72

Litchfield, S. J., Robson, E. I., \& Hughes, D. H. 1995, A\&A, 300, 385

Maoz, D., \& Netzer, H. 1989, MNRAS, 236, 21

Newmark, J. S., Holm, A. V., Imhoff, C. L., Oliverson, N. A., Pitts, R. E., \& Sonneborn, G. 1992, IUE Observing Guide, NASA IUE Newsletter, 47, 1

Nichols, J. S., Garhart, M. P., De La Peña, M. D., \& Levay, K. L. 1993, International Ultraviolet Explorer New Spectral Image Processing System Information Manual: Low-Dispersion Data, Version 1.0, CSC/SD-93/6062

Nichols, J. S., \& Linsky, J. L. 1996, AJ, 111, 1

Peterson, B. M., et al. 1991, ApJ, 368, 119

Peterson, B. M., \& Wandel, A. 1999, ApJ, 521, L95

Peterson, B. M., \& Wandel, A. 2000, ApJ, 540, L13

Peterson, B. M., Wanders, I., Horne, K., Collier, S., Alexander, T., Kaspi, S., \& Maoz, D. 1998, PASP, 110, 660

Reichert, G. A., et al. 1994, ApJ, 425, 582
Rodríguez-Pascual, P. M., et al. 1997, ApJS, 110, 9

Stirpe, G. M., et al. 1994, ApJ, 425, 609

Theureau, G., Bottinelli, L., Coudreau-Durand, N., Gouguenheim, L., Hallet, N., Loulergue, M., Paturel, G., \& Teerikorpi, P. 1998, A\&AS, 130, 333

van Groningen, E., \& Wanders, I. 1992, PASP, 104,700

Wandel, A., Peterson, B. M., \& Malkan, M. A. 1999, ApJ, 526, 579

Wanders, I., et al. 1997, ApJS, 113, 69

White, R. J., \& Peterson, B. M. 1994, PASP, 106, 879

This 2-column preprint was prepared with the AAS IATEX macros v5.0. 


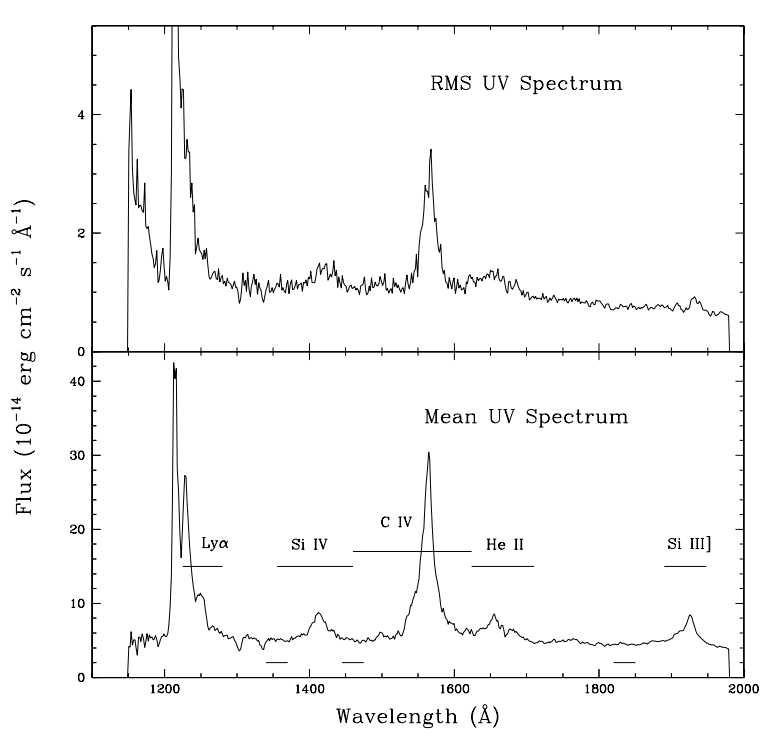

Fig. 1.- Top: RMS UV spectrum. Bottom: Mean UV spectrum. Wavelengths delineated above the spectrum indicate emission-line ranges; those below the spectrum mark ranges of continuum flux measurement.

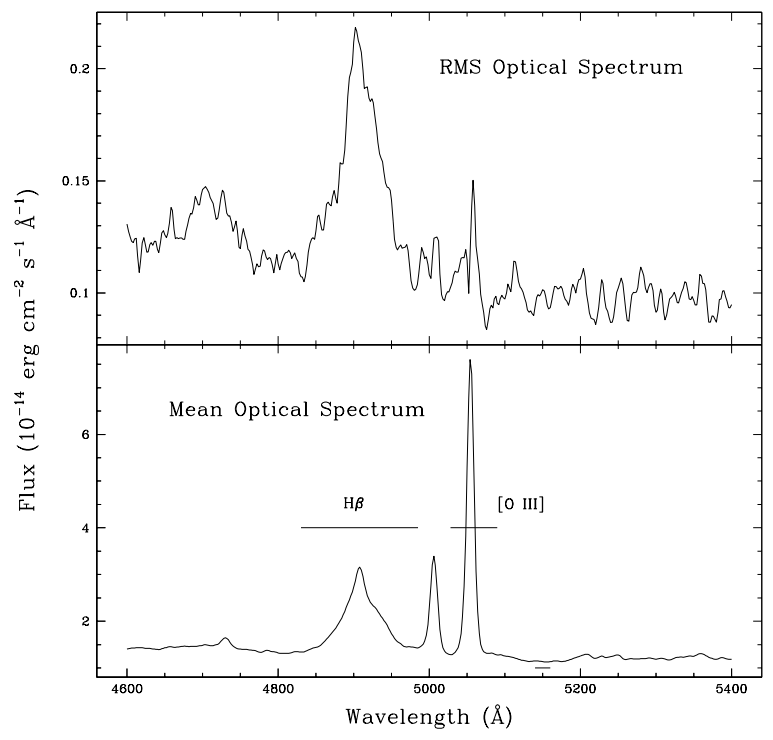

Fig. 2.- Top: RMS optical spectrum. Bottom: Mean optical spectrum. Wavelength indications as in Fig. 1.

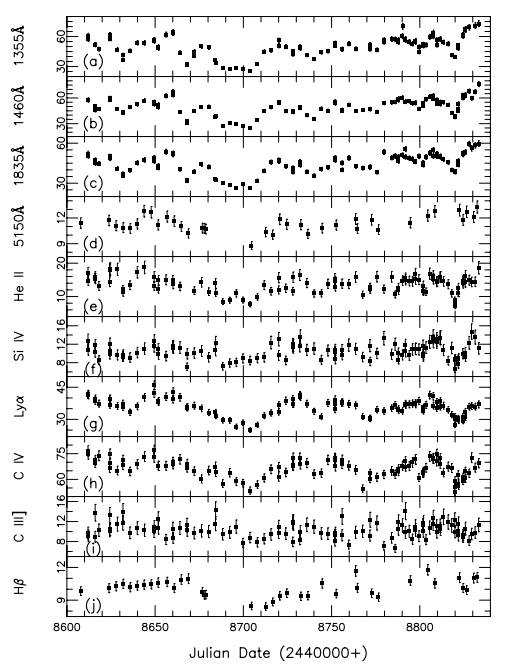

Fig. 3.- UV and optical light curves: (a) 1355 $\AA$ continuum, (b) $1460 \AA$ continuum, (c) $1835 \AA$ continuum, (d) $5150 \AA$ continuum, (e) He II $\lambda 1640$ + O III $\lambda 1663$, (f) Si IV $\lambda 1400+$ O IV] $\lambda 1402$, (g) Ly $\alpha$, (h) C IV $\lambda 1549$, (i) Si III] $\lambda 1892+$ C III] $\lambda 1909$, and (j) $\mathrm{H} \beta$. Continuum fluxes are in units of $10^{-15} \mathrm{ergs} \mathrm{cm}^{-2} \mathrm{~s}^{-1} \AA^{-1}$. Emission line fluxes are given in units of $10^{-13} \mathrm{ergs} \mathrm{cm}^{-2} \mathrm{~s}^{-1}$. 


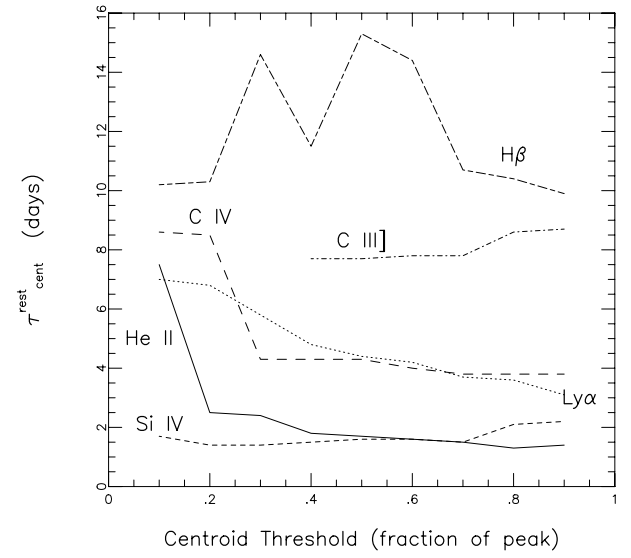

Fig. 4.- Rest-frame centroid time lag versus threshold level for centroid determination (as a fraction of the peak correlation coefficient). The data plotted are for He II $\lambda 1640+$ O III $] \lambda 1663$ (solid), Si IV $\lambda 1400+$ O IV] $\lambda 1402$ (short dashed), Ly $\alpha$ (dotted), C IV $\lambda 1549$ (long dashed), Si III] $\lambda 1892+\mathrm{C}$ III $] \lambda 1909$ (dot-short dashed), and $\mathrm{H} \beta$ (dot-long dashed).

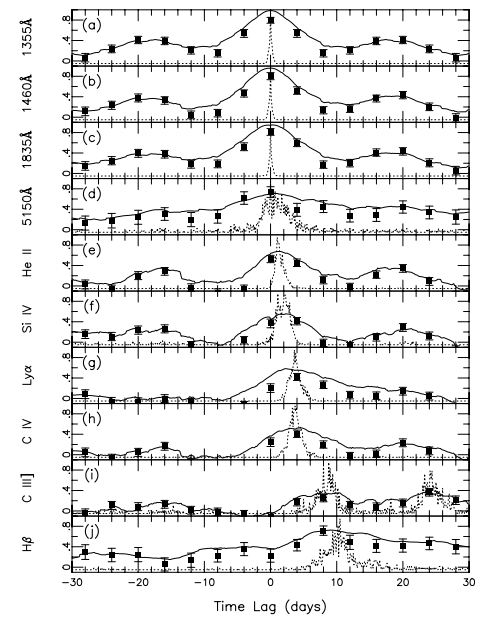

Fig. 5.- Results of cross-correlation of the $1355 \AA$ continuum with (a) itself; (b) $1460 \AA$ continuum; (c) $1835 \AA$ continuum; (d) $5150 \AA$ continuum; (e) He II $\lambda 1640+$ O III $\lambda 1663$; (f) Si IV $\lambda 1400+$ O IV] $\lambda 1402$; (g) Ly $\alpha$; (h) C IV $\lambda 1549$; (i) Si III] $\lambda 1892+\mathrm{C}$ III $] \lambda 1909 ;$ (j) H $\beta$. The solid lines show the ICCFs, the data points are the DCFs, and the dashed lines represent the CCPDs. Note that the $\mathrm{y}$-axis scale for the CCPDs is the fraction of MC realizations producing a centroid of that lag value and is scaled to the maximum in each panel.

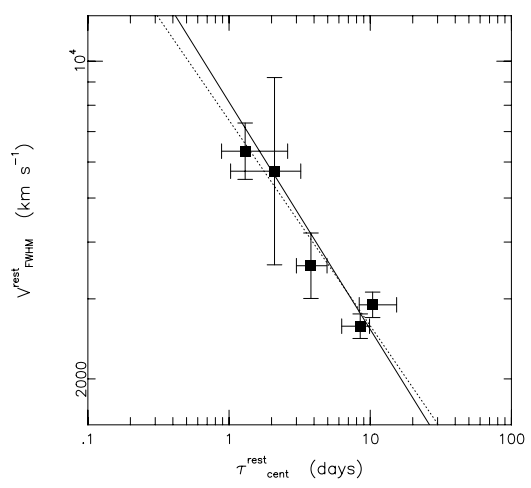

Fig. 6.- Rest-frame velocity FWHM versus restframe centroid time lag for the five emission lines we have measured. The dashed line is the best fit to the data; the solid line is the best fit with fixed slope of -0.5 . 
TABle 1

WAVELENGTH Limits

\begin{tabular}{lc}
\hline \hline \multicolumn{1}{c}{ Line/Band } & Wavelength Range $(\AA)$ \\
\hline $1355 \AA$ continuum & $1340-1370$ \\
$1460 \AA$ continuum & $1445-1475$ \\
$1835 \AA$ continuum & $1820-1850$ \\
$5150 \AA$ continuum & $5140-5160$ \\
Ly $\alpha$ & $1225-1280$ \\
Si IV $\lambda 1400+$ O IV] $\lambda 1402$ & $1355-1460$ \\
C IV $\lambda 1549$ & $1460-1624$ \\
He II $\lambda 1640+$ O III] $\lambda 1663$ & $1624-1710$ \\
Si III $] \lambda 1892+$ C III] $\lambda 1909$ & $1890-1948$ \\
H $\beta$ & $4830-4985$ \\
\hline
\end{tabular}


TABLE 2

UV Continuum Flux Data ${ }^{\mathrm{a}}$

\begin{tabular}{|c|c|c|c|c|}
\hline $\begin{array}{l}\text { Image } \\
\text { Name }\end{array}$ & $\begin{array}{c}\text { Julian Date } \\
(2,440,000+)\end{array}$ & $\mathrm{F}(\lambda 1355)$ & $\mathrm{F}(\lambda 1460)$ & $\mathrm{F}(\lambda 1835)$ \\
\hline SWP 43438 & 8611.948 & $60.811 \pm 2.432$ & $57.899 \pm 2.142$ & $52.244 \pm 1.776$ \\
\hline SWP 43439 & 8612.031 & $57.353 \pm 2.294$ & $57.395 \pm 2.124$ & $50.582 \pm 1.720$ \\
\hline SWP 43472 & 8615.949 & $51.984 \pm 2.079$ & $50.204 \pm 1.858$ & $47.259 \pm 1.607$ \\
\hline SWP 43473 & 8616.029 & $51.979 \pm 2.079$ & $45.407 \pm 1.680$ & $45.024 \pm 1.531$ \\
\hline SWP 43485 & 8618.118 & $47.391 \pm 1.896$ & $47.043 \pm 1.741$ & $44.713 \pm 1.520$ \\
\hline SWP 43539 & 8624.202 & $60.846 \pm 2.434$ & $58.123 \pm 2.151$ & $48.605 \pm 1.653$ \\
\hline SWP 43540 & 8624.294 & $61.371 \pm 2.455$ & $58.173 \pm 2.152$ & $50.031 \pm 1.701$ \\
\hline SWP 43541 & 8624.385 & $58.033 \pm 2.321$ & $59.779 \pm 2.212$ & $48.735 \pm 1.657$ \\
\hline SWP 43557 & 8628.595 & $46.814 \pm 1.873$ & $47.146 \pm 1.744$ & $40.850 \pm 1.389$ \\
\hline SWP 43587 & 8631.680 & $36.975 \pm 1.479$ & $43.277 \pm 1.601$ & $37.136 \pm 1.263$ \\
\hline SWP 43588 & 8631.765 & $41.661 \pm 1.666$ & $42.163 \pm 1.560$ & $35.594 \pm 1.210$ \\
\hline SWP 43636 & 8635.688 & $45.680 \pm 1.827$ & $49.405 \pm 1.828$ & $39.628 \pm 1.347$ \\
\hline SWP 43676 & 8639.862 & $53.819 \pm 2.153$ & $52.490 \pm 1.942$ & $45.264 \pm 1.539$ \\
\hline SWP 43716 & 8643.948 & $53.593 \pm 2.144$ & $56.866 \pm 2.104$ & $47.434 \pm 1.613$ \\
\hline SWP 43871 & 8649.367 & $56.758 \pm 2.270$ & $55.327 \pm 2.047$ & $48.963 \pm 1.665$ \\
\hline SWP 43872 & 8649.454 & $55.680 \pm 2.227$ & $52.634 \pm 1.947$ & $46.580 \pm 1.584$ \\
\hline SWP 43894 & 8651.756 & $49.667 \pm 1.987$ & $48.990 \pm 1.813$ & $43.264 \pm 1.471$ \\
\hline SWP 43895 & 8651.855 & $48.352 \pm 1.934$ & $52.337 \pm 1.936$ & $41.435 \pm 1.409$ \\
\hline SWP 43921 & 8656.130 & $61.961 \pm 2.478$ & $62.436 \pm 2.310$ & $53.664 \pm 1.825$ \\
\hline SWP 43945 & 8660.040 & $64.188 \pm 2.568$ & $67.311 \pm 2.491$ & $51.531 \pm 1.752$ \\
\hline SWP 43946 & 8660.121 & $63.656 \pm 2.546$ & $63.607 \pm 2.353$ & $52.300 \pm 1.778$ \\
\hline SWP 43962 & 8664.048 & $43.983 \pm 1.759$ & $43.716 \pm 1.617$ & $40.467 \pm 1.376$ \\
\hline SWP 43995 & 8668.022 & $32.648 \pm 1.306$ & $30.813 \pm 1.140$ & $31.508 \pm 1.071$ \\
\hline SWP 43996 & 8668.115 & $31.491 \pm 1.260$ & $32.760 \pm 1.212$ & $32.328 \pm 1.099$ \\
\hline SWP 44020 & 8672.106 & $40.887 \pm 1.635$ & $44.671 \pm 1.653$ & $38.755 \pm 1.318$ \\
\hline SWP 44048 & 8676.272 & $44.897 \pm 1.796$ & $49.399 \pm 1.828$ & $44.148 \pm 1.501$ \\
\hline SWP 44072 & 8680.310 & $50.035 \pm 2.001$ & $49.829 \pm 1.844$ & $42.224 \pm 1.436$ \\
\hline SWP 44099 & 8684.199 & $49.072 \pm 1.963$ & $38.191 \pm 1.413$ & $33.057 \pm 1.124$ \\
\hline SWP 44100 & 8684.275 & $36.331 \pm 1.453$ & $39.336 \pm 1.455$ & $32.631 \pm 1.109$ \\
\hline SWP 44126 & 8688.228 & $33.912 \pm 1.356$ & $27.773 \pm 1.028$ & $30.118 \pm 1.024$ \\
\hline SWP 44149 & 8692.216 & $28.542 \pm 1.142$ & $30.410 \pm 1.125$ & $28.579 \pm 0.972$ \\
\hline SWP 44176 & 8695.910 & $27.667 \pm 1.107$ & $29.197 \pm 1.080$ & $26.109 \pm 0.888$ \\
\hline SWP 44189 & 8699.713 & $28.465 \pm 1.139$ & $27.397 \pm 1.014$ & $29.131 \pm 0.990$ \\
\hline SWP 44208 & 8703.723 & $27.708 \pm 1.108$ & $24.374 \pm 0.902$ & $26.402 \pm 0.898$ \\
\hline SWP 44237 & 8707.871 & $25.508 \pm 1.020$ & $33.736 \pm 1.248$ & $31.970 \pm 1.087$ \\
\hline SWP 44267 & 8711.731 & $32.335 \pm 1.293$ & $45.001 \pm 1.665$ & $39.240 \pm 1.334$ \\
\hline SWP 44307 & 8715.612 & $44.933 \pm 1.797$ & $49.245 \pm 1.822$ & $45.781 \pm 1.557$ \\
\hline SWP 44349 & 8719.932 & $46.150 \pm 1.846$ & $55.077 \pm 2.038$ & $47.230 \pm 1.606$ \\
\hline SWP 44350 & 8720.020 & $56.100 \pm 2.244$ & $54.303 \pm 2.009$ & $46.392 \pm 1.577$ \\
\hline SWP 44381 & 8724.004 & $50.768 \pm 2.031$ & $44.383 \pm 1.642$ & $42.641 \pm 1.450$ \\
\hline SWP 44408 & 8727.967 & $49.801 \pm 1.992$ & $44.960 \pm 1.664$ & $41.495 \pm 1.411$ \\
\hline
\end{tabular}


TABle 2-Continued

\begin{tabular}{|c|c|c|c|c|}
\hline $\begin{array}{l}\text { Image } \\
\text { Name }\end{array}$ & $\begin{array}{l}\text { Julian Date } \\
(2,440,000+)\end{array}$ & $\mathrm{F}(\lambda 1355)$ & $\mathrm{F}(\lambda 1460)$ & $\mathrm{F}(\lambda 1835)$ \\
\hline SWP 44409 & 8728.068 & $42.096 \pm$ & $43.414=$ & 38.24 \\
\hline SWP 44410 & & & & \\
\hline SWP 44434 & 31.946 & $.086 \pm 1.723$ & & \\
\hline SWP 44435 & 8732.205 & $.680 \pm 1.987$ & $48.115 \pm 1.780$ & \\
\hline SWP 44461 & 8735.946 & $8.231 \pm 1.929$ & $39.557 \pm 1.464$ & $38.632 \pm 1.313$ \\
\hline SWP 44486 & 8739.990 & $1.475 \pm 1.659$ & $35.484 \pm 1.313$ & $35.752 \pm 1.216$ \\
\hline SWP 44492 & 8744.129 & $.318 \pm 1.493$ & $47.640 \pm 1.763$ & $40.569 \pm 1.379$ \\
\hline SWP 44581 & 8747.874 & $.276 \pm 1.771$ & $45.235 \pm 1.674$ & \\
\hline SWP 44627 & 51.845 & & $55.741 \pm 2.062$ & $4 \pm 1.632$ \\
\hline SWP 44628 & 1.950 & & 53. & 1.666 \\
\hline SWP 44629 & & & & 1.611 \\
\hline SWP 44659 & & & & 1.538 \\
\hline SWP 44660 & & & & \\
\hline SWP 44682 & & & & 1.664 \\
\hline SWP 44731 & & & & 1.448 \\
\hline SWP 44760 & & & 02 & 1.404 \\
\hline SWP 44803 & 39 & 94 & .753 & 1.422 \\
\hline SWP 44804 & 72 & & .722 & 1.438 \\
\hline SWP 44830 & (2) & & 44. & .298 \\
\hline SWP 44873 & 7 & 89 & 54. & 1.814 \\
\hline SWP 44907 & & & & 1.672 \\
\hline SWP 44918 & & & 55. & 1.711 \\
\hline SWP 44921 & & & & $=1.693$ \\
\hline SWP 44922 & & & & $=1.583$ \\
\hline SWP 44935 & & & & $=1.732$ \\
\hline SWP 44949 & & & & \pm 1.693 \\
\hline SWP 44950 & & & & $55.578 \pm 1.890$ \\
\hline SWP 44964 & & & & $48.742 \pm 1.657$ \\
\hline SWP 44974 & & & & $48.098 \pm 1.635$ \\
\hline SWP 44992 & & \pm 2.193 & $53.977 \pm 1.997$ & $46.297 \pm 1.574$ \\
\hline SWP 44993 & & & & $46.258 \pm 1.573$ \\
\hline SWP 45010 & 8799.460 & $54.291 \pm 2.172$ & $47.346 \pm 1.752$ & $50.123 \pm 1.704$ \\
\hline SWP 45024 & & & $50.718 \pm 1.877$ & $45.454 \pm 1.545$ \\
\hline SWP 45025 & & $49.579 \pm 1.983$ & $54.021 \pm 1.999$ & $43.688 \pm 1.485$ \\
\hline SWP 45026 & & & $52.914 \pm 1.958$ & $44.756 \pm 1.522$ \\
\hline SWP 45038 & 03.458 & $52.139 \pm 2.086$ & $58.489 \pm 2.164$ & $49.668 \pm 1.689$ \\
\hline SWP 45052 & 8805.543 & $50.742 \pm 2.030$ & $61.563 \pm 2.278$ & $52.663 \pm 1.791$ \\
\hline SWP 45063 & & $51.641 \pm 2.066$ & $59.254 \pm 2.192$ & $50.333 \pm 1.711$ \\
\hline SWP 45064 & & $587 \pm 2.063$ & $62.612 \pm 2.317$ & $54.971 \pm 1.869$ \\
\hline SWP 45081 & 8809.509 & $59.759 \pm 2.390$ & $57.843 \pm 2.140$ & $50.158 \pm 1.705$ \\
\hline SWP 45082 & 8809.601 & $60.228 \pm 2.409$ & $53.138 \pm 1.966$ & $51.993 \pm 1.768$ \\
\hline
\end{tabular}


TABLE 2-Continued

\begin{tabular}{ccccc}
\hline \hline $\begin{array}{c}\text { Image } \\
\text { Name }\end{array}$ & $\begin{array}{c}\text { Julian Date } \\
(2,440,000+)\end{array}$ & $\mathrm{F}(\lambda 1355)$ & $\mathrm{F}(\lambda 1460)$ & $\mathrm{F}(\lambda 1835)$ \\
\hline SWP 45096 & 8811.493 & $63.860 \pm 2.554$ & $55.395 \pm 2.050$ & $49.903 \pm 1.697$ \\
SWP 45097 & 8811.595 & $56.369 \pm 2.255$ & $52.657 \pm 1.948$ & $50.072 \pm 1.702$ \\
SWP 45106 & 8813.384 & $51.571 \pm 2.063$ & $54.998 \pm 2.035$ & $47.810 \pm 1.626$ \\
SWP 45118 & 8816.028 & $57.179 \pm 2.287$ & $51.851 \pm 1.918$ & $45.563 \pm 1.549$ \\
SWP 45133 & 8818.024 & $57.305 \pm 2.292$ & $42.796 \pm 1.583$ & $39.882 \pm 1.356$ \\
SWP 45150 & 8819.700 & $54.163 \pm 2.167$ & $37.296 \pm 1.380$ & $\ldots$ \\
SWP 45151 & 8819.800 & $52.810 \pm 2.112$ & $38.618 \pm 1.429$ & $38.881 \pm 1.322$ \\
SWP 45152 & 8819.904 & $41.373 \pm 1.655$ & $37.208 \pm 1.377$ & $38.237 \pm 1.300$ \\
SWP 45167 & 8821.689 & $39.568 \pm 1.583$ & $45.306 \pm 1.676$ & $44.545 \pm 1.515$ \\
SWP 45168 & 8821.791 & $48.325 \pm 1.933$ & $47.669 \pm 1.764$ & $46.635 \pm 1.586$ \\
SWP 45169 & 8821.892 & $51.088 \pm 2.044$ & $53.395 \pm 1.976$ & $43.804 \pm 1.489$ \\
SWP 45194 & 8824.353 & $50.394 \pm 2.016$ & $63.151 \pm 2.337$ & $52.316 \pm 1.779$ \\
SWP 45195 & 8824.440 & $61.519 \pm 2.461$ & $60.085 \pm 2.223$ & $51.566 \pm 1.753$ \\
SWP 45206 & 8825.701 & $60.748 \pm 2.430$ & $63.864 \pm 2.363$ & $\cdots$ \\
SWP 45207 & 8825.798 & $66.760 \pm 2.670$ & $62.632 \pm 2.317$ & $53.268 \pm 1.811$ \\
SWP 45219 & 8827.904 & $67.384 \pm 2.695$ & $67.817 \pm 2.509$ & $59.430 \pm 2.021$ \\
SWP 45227 & 8829.302 & $69.254 \pm 2.770$ & $59.651 \pm 2.207$ & $56.059 \pm 1.906$ \\
SWP 45237 & 8831.317 & $70.910 \pm 2.836$ & $67.452 \pm 2.496$ & $58.335 \pm 1.983$ \\
SWP 45246 & 8833.326 & $72.855 \pm 2.914$ & $76.033 \pm 2.813$ & $59.754 \pm 2.032$ \\
\hline
\end{tabular}

${ }^{\mathrm{a}}$ Continuum fluxes are given in units of $10^{-15} \mathrm{ergs} \mathrm{cm}^{-2} \mathrm{~s}^{-1} \AA^{-1}$. 
TABLE 3

UV Emission Line Flux Data ${ }^{a}$

\begin{tabular}{|c|c|c|c|c|c|c|}
\hline $\begin{array}{l}\text { Image } \\
\text { Name }\end{array}$ & $\begin{array}{c}\text { Julian Date } \\
(2,440,000+)\end{array}$ & $\begin{array}{c}\text { He II } \lambda 1640+ \\
\text { O III] } \lambda 1663\end{array}$ & $\begin{array}{c}\text { Si IV } \lambda 1400+ \\
\text { O IV] } \lambda 1402\end{array}$ & $\operatorname{Ly} \alpha$ & C IV $\lambda 1549$ & $\begin{array}{l}\text { Si III] } \lambda 1892+ \\
\quad \text { C III] } \lambda 1909\end{array}$ \\
\hline SWP 43438 & 8611.948 & $17.051 \pm 1.705$ & $12.769 \pm 1.430$ & $41.751 \pm 1.837$ & $76.614 \pm 2.988$ & $9.707 \pm 1.175$ \\
\hline SWP 43439 & 8612.031 & $14.790 \pm 1.479$ & $10.856 \pm 1.216$ & $41.387 \pm 1.821$ & $74.662 \pm 2.912$ & $9.180 \pm 1.111$ \\
\hline SWP 43472 & 8615.949 & $15.837 \pm 1.584$ & $10.362 \pm 1.161$ & $38.720 \pm 1.704$ & $69.633 \pm 2.716$ & $8.968 \pm 1.085$ \\
\hline SWP 43473 & 8616.029 & $15.365 \pm 1.536$ & $11.732 \pm 1.314$ & $39.614 \pm 1.743$ & $71.235 \pm 2.778$ & $13.764 \pm 1.665$ \\
\hline SWP 43485 & 8618.118 & $13.188 \pm 1.319$ & $8.559 \pm 0.959$ & $37.369 \pm 1.644$ & $73.775 \pm 2.877$ & $10.270 \pm 1.243$ \\
\hline SWP 43539 & 8624.202 & $14.384 \pm 1.438$ & $10.623 \pm 1.190$ & $39.431 \pm 1.735$ & $66.575 \pm 2.596$ & $10.175 \pm 1.231$ \\
\hline SWP 43540 & 8624.294 & $17.992 \pm 1.799$ & $9.900 \pm 1.109$ & $36.819 \pm 1.620$ & $69.660 \pm 2.717$ & $13.212 \pm 1.599$ \\
\hline SWP 43541 & 8624.385 & $18.288 \pm 1.829$ & $12.007 \pm 1.345$ & $39.643 \pm 1.744$ & $74.851 \pm 2.919$ & $10.466 \pm 1.266$ \\
\hline SWP 43557 & 8628.595 & $18.169 \pm 1.817$ & $9.672 \pm 1.083$ & $36.259 \pm 1.595$ & $65.172 \pm 2.542$ & $11.489 \pm 1.390$ \\
\hline SWP 43587 & 8631.680 & $12.129 \pm 1.213$ & $9.326 \pm 1.045$ & $37.353 \pm 1.644$ & $68.481 \pm 2.671$ & $11.690 \pm 1.414$ \\
\hline SWP 43588 & 8631.765 & $11.233 \pm 1.123$ & $9.692 \pm 1.086$ & $35.715 \pm 1.571$ & $71.312 \pm 2.781$ & $13.907 \pm 1.683$ \\
\hline SWP 43636 & 8635.688 & $13.382 \pm 1.338$ & $9.124 \pm 1.022$ & $33.320 \pm 1.466$ & $64.623 \pm 2.520$ & $9.733 \pm 1.178$ \\
\hline SWP 43676 & 8639.862 & $17.282 \pm 1.728$ & $10.188 \pm 1.141$ & $36.059 \pm 1.587$ & $68.877 \pm 2.686$ & $10.664 \pm 1.290$ \\
\hline SWP 43716 & 8643.948 & $18.714 \pm 1.871$ & $11.037 \pm 1.236$ & $40.307 \pm 1.774$ & $73.296 \pm 2.859$ & $10.240 \pm 1.239$ \\
\hline SWP 43871 & 8649.367 & $13.185 \pm 1.319$ & $11.574 \pm 1.296$ & $42.142 \pm 1.854$ & $72.814 \pm 2.840$ & $10.015 \pm 1.212$ \\
\hline SWP 43872 & 8649.454 & $15.884 \pm 1.588$ & $12.645 \pm 1.416$ & $45.933 \pm 2.021$ & $76.959 \pm 3.001$ & $10.892 \pm 1.318$ \\
\hline SWP 43894 & 8651.756 & $12.264 \pm 1.226$ & $10.932 \pm 1.224$ & $38.313 \pm 1.686$ & $67.904 \pm 2.648$ & $12.443 \pm 1.506$ \\
\hline SWP 43895 & 8651.855 & $14.863 \pm 1.486$ & $9.827 \pm 1.101$ & $38.525 \pm 1.695$ & $67.918 \pm 2.649$ & $10.770 \pm 1.303$ \\
\hline SWP 43921 & 8656.130 & $15.008 \pm 1.501$ & $9.425 \pm 1.056$ & $40.596 \pm 1.786$ & $67.940 \pm 2.650$ & $8.425 \pm 1.019$ \\
\hline SWP 43945 & 8660.040 & $14.856 \pm 1.486$ & $11.559 \pm 1.295$ & $39.347 \pm 1.731$ & $70.191 \pm 2.737$ & $10.595 \pm 1.282$ \\
\hline SWP 43946 & 8660.121 & $13.709 \pm 1.371$ & $11.282 \pm 1.264$ & $42.857 \pm 1.886$ & $68.775 \pm 2.682$ & $8.694 \pm 1.052$ \\
\hline SWP 43962 & 8664.048 & $13.022 \pm 1.302$ & $11.275 \pm 1.263$ & $40.307 \pm 1.774$ & $71.699 \pm 2.796$ & $10.706 \pm 1.295$ \\
\hline SWP 43995 & 8668.022 & $\ldots$ & $7.140 \pm 0.800$ & $35.489 \pm 1.562$ & $66.840 \pm 2.607$ & $11.294 \pm 1.367$ \\
\hline SWP 43996 & 8668.115 & $\cdots$ & $9.901 \pm 1.109$ & $35.812 \pm 1.576$ & $69.053 \pm 2.693$ & $10.296 \pm 1.246$ \\
\hline SWP 44020 & 8672.106 & $11.764 \pm 1.176$ & $10.083 \pm 1.129$ & $36.446 \pm 1.604$ & $64.753 \pm 2.525$ & $9.706 \pm 1.174$ \\
\hline SWP 44048 & 8676.272 & $14.299 \pm 1.430$ & $10.509 \pm 1.177$ & $35.112 \pm 1.545$ & $60.287 \pm 2.351$ & $10.137 \pm 1.227$ \\
\hline SWP 44072 & 8680.310 & $11.451 \pm 1.145$ & $9.171 \pm 1.027$ & $33.214 \pm 1.461$ & $65.161 \pm 2.541$ & $9.986 \pm 1.208$ \\
\hline SWP 44099 & 8684.199 & $13.925 \pm 1.393$ & $10.682 \pm 1.196$ & $33.220 \pm 1.462$ & $67.461 \pm 2.631$ & $11.558 \pm 1.399$ \\
\hline SWP 44100 & 8684.275 & $11.177 \pm 1.118$ & $12.329 \pm 1.381$ & $31.722 \pm 1.396$ & $64.203 \pm 2.504$ & $14.211 \pm 1.720$ \\
\hline SWP 44126 & 8688.228 & $8.477 \pm 0.848$ & $7.211 \pm 0.808$ & $29.611 \pm 1.303$ & $57.594 \pm 2.246$ & $9.462 \pm 1.145$ \\
\hline SWP 44149 & 8692.216 & $8.912 \pm 0.891$ & $7.930 \pm 0.888$ & $29.519 \pm 1.299$ & $63.911 \pm 2.493$ & $9.846 \pm 1.191$ \\
\hline SWP 44176 & 8695.910 & $10.943 \pm 1.094$ & $8.069 \pm 0.904$ & $26.618 \pm 1.171$ & $58.785 \pm 2.293$ & $10.981 \pm 1.329$ \\
\hline SWP 44189 & 8699.713 & $8.945 \pm 0.894$ & $8.916 \pm 0.999$ & $28.493 \pm 1.254$ & $57.753 \pm 2.252$ & $7.723 \pm 0.934$ \\
\hline SWP 44208 & 8703.723 & $7.663 \pm 0.766$ & $8.471 \pm 0.949$ & $24.982 \pm 1.099$ & $53.504 \pm 2.087$ & $8.793 \pm 1.064$ \\
\hline SWP 44237 & 8707.871 & $9.909 \pm 0.991$ & $8.974 \pm 1.005$ & $27.480 \pm 1.209$ & $56.975 \pm 2.222$ & $7.860 \pm 0.951$ \\
\hline SWP 44267 & 8711.731 & $13.391 \pm 1.339$ & $9.154 \pm 1.025$ & $31.722 \pm 1.396$ & $60.820 \pm 2.372$ & $8.547 \pm 1.034$ \\
\hline SWP 44307 & 8715.612 & $11.593 \pm 1.159$ & $12.355 \pm 1.384$ & $33.015 \pm 1.453$ & $65.884 \pm 2.569$ & $9.412 \pm 1.139$ \\
\hline SWP 44349 & 8719.932 & $11.976 \pm 1.198$ & $9.588 \pm 1.074$ & $33.504 \pm 1.474$ & $61.410 \pm 2.395$ & $9.790 \pm 1.185$ \\
\hline SWP 44350 & 8720.020 & $15.663 \pm 1.566$ & $13.091 \pm 1.466$ & $36.571 \pm 1.609$ & $67.330 \pm 2.626$ & $10.934 \pm 1.323$ \\
\hline SWP 44381 & 8724.004 & $12.488 \pm 1.249$ & $8.475 \pm 0.949$ & $38.718 \pm 1.704$ & $64.045 \pm 2.498$ & $9.589 \pm 1.160$ \\
\hline SWP 44408 & 8727.967 & $11.592 \pm 1.159$ & $11.942 \pm 1.338$ & $39.422 \pm 1.735$ & $68.315 \pm 2.664$ & $7.924 \pm 0.959$ \\
\hline SWP 44409 & 8728.068 & $\ldots$ & $11.346 \pm 1.271$ & $37.210 \pm 1.637$ & $67.746 \pm 2.642$ & $11.943 \pm 1.445$ \\
\hline SWP 44410 & 8728.168 & $15.773 \pm 1.577$ & $11.716 \pm 1.312$ & $37.375 \pm 1.645$ & $72.573 \pm 2.830$ & $11.679 \pm 1.413$ \\
\hline SWP 44434 & 8731.946 & $16.459 \pm 1.646$ & $11.614 \pm 1.301$ & $41.213 \pm 1.813$ & $72.594 \pm 2.831$ & $8.378 \pm 1.014$ \\
\hline SWP 44435 & 8732.205 & $\ldots$ & $13.598 \pm 1.523$ & $40.652 \pm 1.789$ & $68.736 \pm 2.681$ & $9.980 \pm 1.208$ \\
\hline SWP 44461 & 8735.946 & $14.037 \pm 1.404$ & $10.790 \pm 1.208$ & $37.320 \pm 1.642$ & $69.492 \pm 2.710$ & $11.347 \pm 1.373$ \\
\hline SWP 44486 & 8739.990 & $10.913 \pm 1.091$ & $11.056 \pm 1.238$ & $33.716 \pm 1.484$ & $63.079 \pm 2.460$ & $10.973 \pm 1.328$ \\
\hline SWP 44492 & 8744.129 & $10.972 \pm 1.097$ & $8.646 \pm 0.968$ & $31.276 \pm 1.376$ & $63.631 \pm 2.482$ & $9.638 \pm 1.166$ \\
\hline SWP 44581 & 8747.874 & $13.851 \pm 1.385$ & $10.644 \pm 1.192$ & $37.659 \pm 1.657$ & $71.354 \pm 2.783$ & $9.569 \pm 1.158$ \\
\hline SWP 44627 & 8751.845 & $15.350 \pm 1.535$ & $10.311 \pm 1.155$ & $34.631 \pm 1.524$ & $72.491 \pm 2.827$ & $8.062 \pm 0.976$ \\
\hline SWP 44628 & 8751.950 & $14.578 \pm 1.458$ & $10.779 \pm 1.207$ & $39.274 \pm 1.728$ & $71.496 \pm 2.788$ & $9.285 \pm 1.123$ \\
\hline SWP 44629 & 8752.056 & $12.920 \pm 1.292$ & $8.708 \pm 0.975$ & $36.988 \pm 1.627$ & $67.524 \pm 2.633$ & $9.705 \pm 1.174$ \\
\hline SWP 44659 & 8755.872 & $13.868 \pm 1.387$ & $10.749 \pm 1.204$ & $37.794 \pm 1.663$ & $68.993 \pm 2.691$ & $9.236 \pm 1.118$ \\
\hline
\end{tabular}


TABle 3-Continued

\begin{tabular}{|c|c|c|c|c|c|c|}
\hline $\begin{array}{l}\text { Image } \\
\text { Name }\end{array}$ & $\begin{array}{c}\text { Julian Date } \\
(2,440,000+)\end{array}$ & $\begin{array}{c}\text { He II } \lambda 1640+ \\
\text { O III] } \lambda 1663\end{array}$ & $\begin{array}{c}\text { Si IV } \lambda 1400+ \\
\text { O IV] } \lambda 1402\end{array}$ & Ly $\alpha$ & C IV $\lambda 1549$ & $\begin{array}{l}\text { Si III] } \lambda 1892+ \\
\quad \text { C III] } \lambda 1909\end{array}$ \\
\hline SWP 44660 & 8755.949 & & $9.701 \pm 1.087$ & $37.884 \pm 1.667$ & $71.405 \pm 2.785$ & $13.026 \pm 1.576$ \\
\hline SWP 44682 & 8759.702 & $13.730 \pm 1.373$ & $11.811 \pm 1.323$ & $37.249 \pm 1.639$ & $68.033 \pm 2.653$ & $7.400 \pm 0.895$ \\
\hline SWP 44731 & 8763.559 & $15.880 \pm 1.588$ & $10.974 \pm 1.229$ & $36.521 \pm 1.607$ & $65.598 \pm 2.558$ & $9.866 \pm 1.194$ \\
\hline SWP 44760 & 8767.535 & $10.554 \pm 1.055$ & $9.223 \pm 1.033$ & $31.337 \pm 1.379$ & $54.612 \pm 2.130$ & $10.060 \pm 1.217$ \\
\hline SWP 44803 & 8771.689 & $12.725 \pm 1.273$ & $11.678 \pm 1.308$ & $30.261 \pm 1.331$ & $60.436 \pm 2.357$ & $9.198 \pm 1.113$ \\
\hline SWP 44804 & 8771.772 & $12.169 \pm 1.217$ & $8.260 \pm 0.925$ & $30.620 \pm 1.347$ & $63.469 \pm 2.475$ & $12.367 \pm 1.496$ \\
\hline SWP 44830 & 8775.633 & $15.957 \pm 1.596$ & $10.018 \pm 1.122$ & $34.556 \pm 1.520$ & $61.264 \pm 2.389$ & $11.703 \pm 1.416$ \\
\hline SWP 44873 & 8779.607 & $12.230 \pm 1.223$ & $13.309 \pm 1.491$ & $33.902 \pm 1.492$ & $62.857 \pm 2.451$ & $7.113 \pm 0.861$ \\
\hline SWP 44907 & 8783.948 & $15.930 \pm 1.593$ & $9.829 \pm 1.101$ & $35.165 \pm 1.547$ & $65.009 \pm 2.535$ & $8.760 \pm 1.060$ \\
\hline SWP 44918 & 8785.949 & $10.634 \pm 1.063$ & $11.991 \pm 1.343$ & $36.745 \pm 1.617$ & $62.983 \pm 2.456$ & $6.630 \pm 0.802$ \\
\hline SWP 44921 & 8787.786 & $12.702 \pm 1.270$ & $10.055 \pm 1.126$ & $34.865 \pm 1.534$ & $64.779 \pm 2.526$ & $10.548 \pm 1.276$ \\
\hline SWP 44922 & 8787.864 & $11.840 \pm 1.184$ & $8.687 \pm 0.973$ & $34.194 \pm 1.505$ & $66.079 \pm 2.577$ & $11.881 \pm 1.438$ \\
\hline SWP 44935 & 8790.113 & $14.784 \pm 1.478$ & $9.681 \pm 1.084$ & $33.982 \pm 1.495$ & $67.992 \pm 2.652$ & $11.232 \pm 1.359$ \\
\hline SWP 44949 & 8791.769 & $15.495 \pm 1.549$ & $11.760 \pm 1.317$ & $37.262 \pm 1.640$ & $70.532 \pm 2.751$ & $9.822 \pm 1.188$ \\
\hline SWP 44950 & 8791.857 & $15.004 \pm 1.500$ & $10.425 \pm 1.168$ & $36.716 \pm 1.616$ & $67.412 \pm 2.629$ & $14.068 \pm 1.702$ \\
\hline SWP 44964 & 8793.963 & $14.658 \pm 1.466$ & $9.572 \pm 1.072$ & $37.016 \pm 1.629$ & $67.780 \pm 2.643$ & $10.045 \pm 1.215$ \\
\hline SWP 44974 & 8795.768 & $14.577 \pm 1.458$ & $10.878 \pm 1.218$ & $37.405 \pm 1.646$ & $69.919 \pm 2.727$ & $8.464 \pm 1.024$ \\
\hline SWP 44992 & 8797.448 & $16.789 \pm 1.679$ & $11.013 \pm 1.233$ & $37.606 \pm 1.655$ & $71.690 \pm 2.796$ & $11.005 \pm 1.332$ \\
\hline SWP 44993 & 8797.538 & $15.144 \pm 1.514$ & $10.938 \pm 1.225$ & $37.047 \pm 1.630$ & $71.722 \pm 2.797$ & $10.318 \pm 1.248$ \\
\hline SWP 45010 & 8799.460 & $15.066 \pm 1.507$ & $10.861 \pm 1.216$ & $38.602 \pm 1.698$ & $61.098 \pm 2.383$ & $9.052 \pm 1.095$ \\
\hline SWP 45024 & 8801.764 & $11.581 \pm 1.158$ & $11.610 \pm 1.300$ & $\ldots$ & $63.585 \pm 2.480$ & $11.248 \pm 1.361$ \\
\hline SWP 45025 & 8801.887 & $13.310 \pm 1.331$ & $9.372 \pm 1.050$ & $34.032 \pm 1.497$ & $64.283 \pm 2.507$ & $10.079 \pm 1.220$ \\
\hline SWP 45026 & 8801.996 & $11.679 \pm 1.168$ & $9.878 \pm 1.106$ & $35.559 \pm 1.565$ & $64.598 \pm 2.519$ & $11.732 \pm 1.420$ \\
\hline SWP 45038 & 8803.458 & $11.433 \pm 1.143$ & $11.278 \pm 1.263$ & $36.813 \pm 1.620$ & $66.819 \pm 2.606$ & $8.374 \pm 1.013$ \\
\hline SWP 45052 & 8805.543 & $16.844 \pm 1.684$ & $12.541 \pm 1.405$ & $41.348 \pm 1.819$ & $73.452 \pm 2.865$ & $10.795 \pm 1.306$ \\
\hline SWP 45063 & 8807.520 & $16.108 \pm 1.611$ & $13.618 \pm 1.525$ & $40.783 \pm 1.794$ & $72.034 \pm 2.809$ & $11.587 \pm 1.402$ \\
\hline SWP 45064 & 8807.603 & $15.292 \pm 1.529$ & $12.634 \pm 1.415$ & $36.449 \pm 1.604$ & $72.370 \pm 2.822$ & $12.714 \pm 1.538$ \\
\hline SWP 45081 & 8809.509 & $14.375 \pm 1.438$ & $12.532 \pm 1.404$ & $35.314 \pm 1.554$ & $70.550 \pm 2.751$ & $11.009 \pm 1.332$ \\
\hline SWP 45082 & 8809.601 & $14.549 \pm 1.455$ & $13.052 \pm 1.462$ & $38.033 \pm 1.673$ & $74.732 \pm 2.915$ & $9.878 \pm 1.195$ \\
\hline SWP 45096 & 8811.493 & $15.791 \pm 1.579$ & $13.392 \pm 1.500$ & $35.964 \pm 1.582$ & $69.099 \pm 2.695$ & $11.158 \pm 1.350$ \\
\hline SWP 45097 & 8811.595 & $15.899 \pm 1.590$ & $13.384 \pm 1.499$ & $38.451 \pm 1.692$ & $72.306 \pm 2.820$ & $10.461 \pm 1.266$ \\
\hline SWP 45106 & 8813.384 & $14.546 \pm 1.455$ & $10.726 \pm 1.201$ & $36.440 \pm 1.603$ & $64.005 \pm 2.496$ & $12.925 \pm 1.564$ \\
\hline SWP 45118 & 8816.028 & $13.700 \pm 1.370$ & $8.430 \pm 0.944$ & $34.493 \pm 1.518$ & $67.487 \pm 2.632$ & $11.906 \pm 1.441$ \\
\hline SWP 45133 & 8818.024 & $10.933 \pm 1.093$ & $11.187 \pm 1.253$ & $32.175 \pm 1.416$ & $66.611 \pm 2.598$ & $11.225 \pm 1.358$ \\
\hline SWP 45150 & 8819.700 & $8.954 \pm 0.895$ & $8.490 \pm 0.951$ & $27.355 \pm 1.204$ & $55.596 \pm 2.168$ & \\
\hline SWP 45151 & 8819.800 & $7.794 \pm 0.779$ & $6.574 \pm 0.736$ & $31.274 \pm 1.376$ & $52.838 \pm 2.061$ & $12.267 \pm 1.484$ \\
\hline SWP 45152 & 8819.904 & $7.073 \pm 0.707$ & $8.813 \pm 0.987$ & $30.107 \pm 1.325$ & $59.321 \pm 2.314$ & $9.929 \pm 1.201$ \\
\hline SWP 45167 & 8821.689 & $11.037 \pm 1.104$ & $8.706 \pm 0.975$ & $30.555 \pm 1.344$ & $62.589 \pm 2.441$ & $10.167 \pm 1.230$ \\
\hline SWP 45168 & 8821.791 & $12.697 \pm 1.270$ & $7.656 \pm 0.857$ & $29.693 \pm 1.306$ & $57.353 \pm 2.237$ & $9.218 \pm 1.115$ \\
\hline SWP 45169 & 8821.892 & $11.109 \pm 1.111$ & $8.925 \pm 1.000$ & $29.510 \pm 1.298$ & $56.358 \pm 2.198$ & $11.512 \pm 1.393$ \\
\hline SWP 45194 & 8824.353 & $14.707 \pm 1.471$ & $9.936 \pm 1.113$ & $31.339 \pm 1.379$ & $59.284 \pm 2.312$ & $8.923 \pm 1.080$ \\
\hline SWP 45195 & 8824.440 & $13.914 \pm 1.391$ & $\ldots$ & $29.242 \pm 1.287$ & $61.297 \pm 2.391$ & $9.275 \pm 1.122$ \\
\hline SWP 45206 & 8825.701 & $14.222 \pm 1.422$ & $10.173 \pm 1.139$ & $33.615 \pm 1.479$ & $64.641 \pm 2.521$ & $\ldots$ \\
\hline SWP 45207 & 8825.798 & $16.205 \pm 1.620$ & $9.322 \pm 1.044$ & $30.904 \pm 1.360$ & $60.589 \pm 2.363$ & $10.583 \pm 1.281$ \\
\hline SWP 45219 & 8827.904 & $14.897 \pm 1.490$ & $12.322 \pm 1.380$ & $35.942 \pm 1.581$ & $63.532 \pm 2.478$ & $8.180 \pm 0.990$ \\
\hline SWP 45227 & 8829.302 & $14.370 \pm 1.437$ & $14.745 \pm 1.651$ & $36.139 \pm 1.590$ & $72.627 \pm 2.832$ & $9.507 \pm 1.150$ \\
\hline SWP 45237 & 8831.317 & $14.427 \pm 1.443$ & $13.534 \pm 1.516$ & $35.966 \pm 1.583$ & $66.867 \pm 2.608$ & $9.803 \pm 1.186$ \\
\hline SWP 45246 & 8833.326 & $18.615 \pm 1.862$ & $11.108 \pm 1.244$ & $36.969 \pm 1.627$ & $69.398 \pm 2.707$ & $11.312 \pm 1.369$ \\
\hline
\end{tabular}

${ }^{\mathrm{a}}$ Emission line fluxes are in units of $10^{-13} \mathrm{ergs}^{-2} \mathrm{~s}^{-1}$. 
TABLE 4

UV VELOCITY DATA ${ }^{\mathrm{a}}$

\begin{tabular}{lcc}
\hline \hline \multicolumn{1}{c}{ Emission Line } & $\mathrm{V}_{F W H M}^{\text {rest }}(\mathrm{rms})$ & $\mathrm{V}_{F W H M}^{\text {rest }}($ mean $)$ \\
\hline He II $\lambda 1640+$ O III] $\lambda 1663$ & $6.34 \pm 0.90$ & $4.74 \pm 0.69$ \\
Si IV $\lambda 1400+$ O IV] $\lambda 1402$ & $5.73 \pm 2.71$ & $4.81 \pm 0.50$ \\
Ly $\alpha$ & $\ldots$ & $\ldots$ \\
C IV $\lambda 1549$ & $3.55 \pm 0.59$ & $3.03 \pm 0.07$ \\
Si III] $\lambda 1892+$ C III] $\lambda 1909$ & $2.61 \pm 0.16$ & $2.82 \pm 0.31$ \\
\hline
\end{tabular}

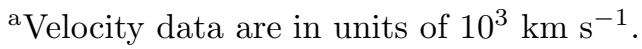


TABLE 5

Optical Flux Data

\begin{tabular}{cccc}
\hline \hline Image & Julian Date & & \\
Name & $(2,440,000+)$ & $\mathrm{F}(\lambda 5150)^{\mathrm{a}}$ & $\mathrm{H} \beta^{\mathrm{b}}$ \\
\hline $\mathrm{n} 38607 \mathrm{a}$ & 8607.830 & $11.406 \pm 0.605$ & $9.829 \pm 0.403$ \\
$\mathrm{n} 38623 \mathrm{a}$ & 8623.830 & $11.707 \pm 0.620$ & $10.100 \pm 0.414$ \\
$\mathrm{n} 38627 \mathrm{a}$ & 8627.830 & $11.094 \pm 0.588$ & $10.272 \pm 0.421$ \\
$\mathrm{n} 38631 \mathrm{a}$ & 8631.840 & $10.865 \pm 0.576$ & $10.494 \pm 0.430$ \\
$\mathrm{n} 38635 \mathrm{a}$ & 8635.830 & $10.781 \pm 0.571$ & $10.183 \pm 0.418$ \\
$\mathrm{n} 38639 \mathrm{a}$ & 8639.840 & $11.303 \pm 0.599$ & $10.327 \pm 0.423$ \\
$\mathrm{n} 38643 \mathrm{a}$ & 8643.720 & $12.788 \pm 0.678$ & $10.423 \pm 0.427$ \\
$\mathrm{n} 38647 \mathrm{a}$ & 8647.760 & $12.668 \pm 0.671$ & $10.459 \pm 0.429$ \\
$\mathrm{n} 38651 \mathrm{a}$ & 8651.670 & $11.190 \pm 0.593$ & $10.603 \pm 0.435$ \\
$\mathrm{n} 38656 \mathrm{a}$ & 8656.770 & $12.163 \pm 0.645$ & $10.669 \pm 0.437$ \\
$\mathrm{n} 38660 \mathrm{a}$ & 8660.770 & $11.629 \pm 0.616$ & $10.109 \pm 0.414$ \\
$\mathrm{n} 38664 \mathrm{a}$ & 8664.770 & $11.005 \pm 0.583$ & $10.894 \pm 0.447$ \\
$\mathrm{n} 38668 \mathrm{a}$ & 8668.810 & $10.204 \pm 0.541$ & $10.969 \pm 0.450$ \\
$\mathrm{n} 38676 \mathrm{a}$ & 8676.710 & $10.817 \pm 0.573$ & $9.760 \pm 0.400$ \\
$\mathrm{n} 38677 \mathrm{a}$ & 8677.800 & $10.786 \pm 0.572$ & $9.483 \pm 0.389$ \\
$\mathrm{n} 38678 \mathrm{a}$ & 8678.680 & $10.703 \pm 0.567$ & $9.513 \pm 0.390$ \\
$\mathrm{n} 38704 \mathrm{a}$ & 8704.580 & $8.763 \pm 0.464$ & $8.469 \pm 0.347$ \\
$\mathrm{n} 38712 \mathrm{a}$ & 8712.590 & $10.350 \pm 0.549$ & $8.410 \pm 0.345$ \\
$\mathrm{n} 38716 \mathrm{a}$ & 8716.600 & $10.055 \pm 0.533$ & $8.840 \pm 0.362$ \\
$\mathrm{n} 38720 \mathrm{a}$ & 8720.590 & $11.842 \pm 0.628$ & $9.395 \pm 0.385$ \\
$\mathrm{n} 38724 \mathrm{a}$ & 8724.560 & $11.257 \pm 0.597$ & $9.640 \pm 0.395$ \\
$\mathrm{n} 38732 \mathrm{a}$ & 8732.560 & $11.212 \pm 0.594$ & $9.357 \pm 0.384$ \\
$\mathrm{n} 38736 \mathrm{a}$ & 8736.550 & $10.149 \pm 0.538$ & $9.410 \pm 0.386$ \\
$\mathrm{n} 38744 \mathrm{a}$ & 8744.610 & $10.812 \pm 0.573$ & $10.537 \pm 0.432$ \\
$\mathrm{n} 38752 \mathrm{a}$ & 8752.570 & $11.174 \pm 0.592$ & $9.606 \pm 0.394$ \\
$\mathrm{n} 38763 \mathrm{a}$ & 8763.580 & $11.880 \pm 0.630$ & $11.678 \pm 0.479$ \\
$\mathrm{n} 38764 \mathrm{a}$ & 8764.560 & $10.754 \pm 0.570$ & $10.131 \pm 0.415$ \\
$\mathrm{n} 38772 \mathrm{a}$ & 8772.630 & $11.799 \pm 0.625$ & $9.627 \pm 0.395$ \\
$\mathrm{n} 38776 \mathrm{a}$ & 8776.560 & $10.636 \pm 0.564$ & $9.272 \pm 0.380$ \\
$\mathrm{n} 38794 \mathrm{a}$ & 8794.570 & $11.444 \pm 0.607$ & $10.803 \pm 0.443$ \\
$\mathrm{n} 38804 \mathrm{a}$ & 8804.470 & $12.281 \pm 0.651$ & $11.780 \pm 0.483$ \\
$\mathrm{n} 38808 \mathrm{a}$ & 8808.480 & $12.773 \pm 0.677$ & $10.531 \pm 0.432$ \\
$\mathrm{n} 38822 \mathrm{a}$ & 8822.480 & $12.917 \pm 0.685$ & $11.060 \pm 0.453$ \\
$\mathrm{n} 38824 \mathrm{a}$ & 8824.470 & $11.765 \pm 0.624$ & $10.108 \pm 0.414$ \\
$\mathrm{n} 38826 \mathrm{a}$ & 8826.470 & $12.717 \pm 0.674$ & $9.912 \pm 0.406$ \\
$\mathrm{n} 38830 \mathrm{a}$ & 8830.480 & $12.108 \pm 0.642$ & $11.026 \pm 0.452$ \\
$\mathrm{n} 38832 \mathrm{a}$ & 8832.460 & $13.281 \pm 0.704$ & $11.086 \pm 0.455$ \\
\hline & & & \\
& & & \\
& & & \\
& & & \\
& &
\end{tabular}

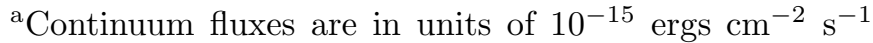
$\AA^{-1}$.

${ }^{\mathrm{b}}$ Emission line fluxes are in units of $10^{-13} \mathrm{ergs} \mathrm{cm}^{-2} \mathrm{~s}^{-1}$.
} 
TABle 6

SAMPLing StATistics

\begin{tabular}{|c|c|c|c|c|c|c|}
\hline \multirow[b]{2}{*}{ Subset } & \multirow[b]{2}{*}{ Number } & \multicolumn{2}{|c|}{ Sampling Interval (days) } & \multirow[b]{2}{*}{$\mathrm{F}_{v a r}$} & \multirow[b]{2}{*}{$\mathrm{R}_{\max }$} & \multirow[b]{2}{*}{ Reference } \\
\hline & & Average & Median & & & \\
\hline Previous $1460 \AA$ continuum dataset & 69 & 3.3 & 3.9 & 0.201 & $3.027 \pm 0.380$ & 1 \\
\hline New UV dataset; binned by epoch & 69 & 3.3 & 3.9 & 0.203 & $3.119 \pm 0.163$ & \\
\hline New UV dataset; complete sample & 101 & 2.2 & 2.0 & 0.192 & $2.856 \pm 0.162$ & \\
\hline New UV dataset; 4-day sampling period & 62 & 2.8 & 3.9 & 0.193 & $2.762 \pm 0.145$ & \\
\hline New UV dataset; 2-day sampling period & 40 & 1.3 & 1.7 & 0.140 & $2.043 \pm 0.107$ & \\
\hline Previous $5150 \AA$ A continuum dataset & 72 & 3.2 & 2.0 & 0.078 & $1.517 \pm 0.042$ & 2 \\
\hline New optical dataset; binned by epoch & 35 & 6.6 & 4.0 & 0.065 & $1.516 \pm 0.114$ & \\
\hline New optical dataset; complete sample & 37 & 6.2 & 4.0 & 0.064 & $1.516 \pm 0.114$ & \\
\hline
\end{tabular}

REFEREnCes.-(1) Reichert et al. 1994; (2) Stirpe et al. 1994.

Note.-Previously published light curves were collected from the AGN Watch website. 
TABLE 7

Cross-Correlation Results ${ }^{\mathrm{a}}$

\begin{tabular}{lrrrr}
\hline \hline & \multicolumn{2}{c}{ Previous Results $^{\mathrm{b}}$} & \multicolumn{2}{c}{ Current Results } \\
\cline { 2 - 5 } \multicolumn{1}{c}{ Line/Band } & \multicolumn{1}{c}{$\tau_{\text {cent }}^{\text {rest }}$} & \multicolumn{1}{c}{$\tau_{\text {peak }}^{\text {rest }}$} & \multicolumn{1}{c}{$\tau_{\text {cent }}^{\text {rest }}$} & \multicolumn{1}{c}{$\tau_{\text {peak }}^{\text {rest }}$} \\
\hline $\mathrm{F}(\lambda 1460)$ & $\ldots$ & $\ldots$ & $-0.1_{-0.2}^{+0.3}$ & $0.0_{-0.4}^{+0.2}$ \\
$\mathrm{~F}(\lambda 1835)$ & $0.1_{-3}^{+3}$ & $0_{-2}^{+2}$ & $0.0_{-0.3}^{+0.3}$ & $0.0_{-0.5}^{+0.6}$ \\
$\mathrm{~F}(\lambda 5150)$ & $1.6_{-2}^{+2}$ & $1_{-2}^{+2}$ & $0.4_{-1.6}^{+3.1}$ & $0.7_{-1.6}^{+1.9}$ \\
He II $\lambda 1640+$ O III] $\lambda 1663$ & $0.5_{-4}^{+4}$ & $1_{-2}^{+2}$ & $1.3_{-0.5}^{+0.9}$ & $1.4_{-1.1}^{+0.6}$ \\
Si IV $\lambda 1400+$ O IV] $\lambda 1402$ & $3.9_{-4}^{+4}$ & $5_{-2}^{+2}$ & $2.1_{-1.5}^{+0.9}$ & $2.3_{-2.4}^{+0.8}$ \\
Ly $\alpha$ & $3.8_{-3}^{+3}$ & $4_{-2}^{+2}$ & $3.6_{-0.7}^{+1.1}$ & $2.2_{-0.1}^{+2.5}$ \\
C IV $\lambda 1549$ & $5.4_{-3}^{+3}$ & $5_{-2}^{+2}$ & $3.8_{-0.9}^{+1.0}$ & $4.5_{-2.2}^{+0.4}$ \\
Si III $\lambda 1892+\mathrm{C} \mathrm{III]} \lambda 1909^{\mathrm{c}}$ & $15.6_{-4}^{+4}$ & $9_{-2}^{+2}$ & $8.5_{-2.6}^{+1.3}$ & $10.2_{-5.3}^{+0.2}$ \\
$\mathrm{H} \beta$ & $7.1_{-2}^{+2}$ & $8_{-2}^{+2}$ & $10.4_{-2.3}^{+4.1}$ & $9.0_{-2.4}^{+5.1}$ \\
\hline
\end{tabular}

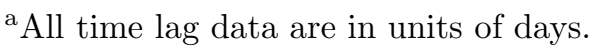

${ }^{\mathrm{b}}$ The previous results listed here are adapted from the GEX-extracted UV data of Reichert et al. (1994) and from the optical results of Stirpe et al. (1994), both of which used the $1460 \AA$ continuum as the driving light curve.

${ }^{\mathrm{c}}$ The range of time lags we included in our analysis of Si III] $\lambda 1892+$ C III] $\lambda 1909$ was limited to \pm 16 days to avoid aliasing. 